\title{
Les conséquences de la réforme sur l'agriculture française
}

Oléagineux, Corps Gras, Lipides. Volume 11, Numéro 4, 301-8, JUILLET-OCTOBRE 2004, CONSÉQUENCES DE LA RÉFORME

\author{
Auteur(s) : Dominique DROUET \\ Centre d'économie rurale de la Mayenne Parc Technopole de changé, Rue Albert Einstein, BP 26116 \\ 53061 Laval cedex 9. Tél. : 0243498400 Fax. : 0243498401
}

Résumé : The fall of the intervention prices on the principal products and their replacement by direct assistances was translated, over the long period since 1992, by falls of result of the exploitations. The decoupling of the assistances, even partial assistances, will change the mode of management reasoning in agricultural firms. The economic result of a production will henceforth be calculated without taking into account the European assistance in the receipt. That mechanically reduces the level of result of this production. It results from it a modification from the relative interest from the choice from such or such agricultural production. That will modify the inputs use, the irrigation and the firm organisation. That will call into question the worse grounds use. It should result from that a strong reorganization from French agriculture and agroalimentary industries. The choice of the French government to maintain, for the bovine nursing herd, the assistances coupled with production will stiffen this production in its volume and on the territory which it currently occupies. The fall of the support prices of milk with the introduction of a compensation subsidy without link with the production will lead to a restructuring of the exploitations, of milk industries and to a redistribution of the production on the territory. Finally, this new European Common Agricultural Policy gets French agriculture in front of the obligation to transform. To some extent, it is found in front of the need for a revision which has the same scope as that of 1962.

Mots-clés : CAP, decoupling, bovine nursing, French agriculture, big farm, single payment

\section{ARTICLE}

Nous ne reviendrons pas sur les causes des évolutions de la PAC qui ont conduit aux réformes successives de Berlin et de Luxembourg. Les causes, les enjeux et le déroulement des négociations ont été abondamment décrits par ailleurs. De même, nous choisissons de ne pas décrire le contenu de la réforme de Luxembourg puisque de multiples articles ont été écrits sur ce sujet. Notre propos se veut une analyse prospective des conséquences de ces réformes sur l'agriculture française à partir d'une prise en compte de ses conséquences sur la réaction et les décisions des agents économiques et, en premier lieu, des agriculteurs et des entreprises d'aval de l'agriculture. 


\section{Les effets de la réforme sur les entreprises agricoles et agro-alimentaires}

\section{Introduction}

Pour étudier les effets de l'accord de Luxembourg sur les entreprises agricoles et agro-alimentaires, il faut aussi prendre en compte la réforme de Berlin (1999) et celle à venir de l'OCM sucre. Elles forment un ensemble construit sur quatre principes : la baisse des prix de soutien; la mise en place d'aides directes ; le découplage de ces aides directes ; l'éco-conditionnalité.

Tout cela permet de rendre la PAC socialement acceptable et compatible avec les contraintes de I'OMC, à l'intérieur d'un budget dont l'enveloppe maximale a été fixée par l'accord Chirac-Schroëder de septembre 2002 pour une période allant jusqu'à 2013. Ce budget inclut le coût de l'application de la PAC aux Peco.

\section{Les effets de la baisse des prix de soutien}

Le lien n'est pas direct entre le prix de soutien et le prix payé au producteur. Mais dans une situation fréquente d'offre excédentaire, la baisse du prix de soutien entraîne une baisse du chiffre d'affaires du producteur, accompagnée d'une plus grande volatilité des prix. Depuis 1992, cela s'est traduit par une baisse du résultat courant en grandes cultures (figures 1 et 2 ).

Il en résulte : une baisse de la marge brute; une plus grande variabilité de la marge. Et cela a pour conséquences dans les décisions de gestion :

- la recherche de compensation de la marge : soit par la baisse des charges; soit par le changement de production; soit par l'augmentation de la taille de l'exploitation.

- - le besoin de dégager en année "normale » une marge de sécurité, qui n'était pas aussi nécessaire auparavant, afin de faire face aux variations de prix.

Il faut souligner que ces effets ne concernent pas seulement les exploitations mais aussi les entreprises de collecte et de transformation. Leur équilibre économique est aussi mis en péril et elles doivent, de ce fait, réorienter leur stratégie.

\section{La place des aides directes}

Depuis 1992 pour les grandes cultures, plus récemment en viande bovine et demain pour la production laitière, les aides directes ont pris une place importante dans les recettes des exploitations agricoles. Aussi faut-il souligner que, dans l'état actuel des systèmes de production au moment de l'accord de Luxembourg, il n'est pas possible de produire, sans les aides directes, le blé, l'orge, le colza, le maïs, les vaches allaitantes, les taurillons et le lait.

\section{Remarques}

- 1. A propos de l'adaptabilité des systèmes: c'est bien dans les systèmes tels qu'ils sont aujourd'hui que les aides sont indispensables pour produire. Ces systèmes résultent de l'adaptation au contexte économique des dernières années au cours desquelles ces aides ont été distribuées. Dans un autre contexte, il est probable que les équilibres entre les charges et les produits auraient été établis différemment avec une autre combinaison des facteurs de 
production. On peut à la fois imaginer que d'autres systèmes de production rentables soient possibles et que, pour certaines productions et sur certains territoires, il n'existe aucune issue avec moins d'aides européennes.

- 2. L'effet de la modulation : la modulation va venir amputer les aides de $5 \%$. Ce montant sera accru du niveau de la réserve nationale, au maximum $3 \%$. Par ailleurs, une dégressivité des aides ne peut être exclue si le budget de l'Europe se révélait insuffisant d'ici 2013. En fait, à l'avenir, il faut prendre en compte une baisse des aides de l'ordre de 7 à $8 \%$. Ceci représente une amputation notable des revenus, toutes choses égales par ailleurs. Cette diminution aura pour conséquence d'accroître la rapidité des changements.

\section{Le découplage et ses effets}

On appelle « découplage " l'attribution à l'exploitant agricole d'une aide directe appelée « paiement unique » qui est déliée de tout acte et/ou de tout choix de production. Le bénéfice du "droit à paiement unique " DPU est seulement soumis à l'obligation d'entretenir les terres correspondantes dans le respect d'un certain nombre de critères relevant du dispositif dit " d'éco-conditionnalité ». Le DPU est calculé sur la moyenne des aides directes perçues par l'exploitation sur les trois années 2000-2001-2002. En définitive, on peut considérer l'aide découplée comme un " produit fixe " qui vient financer l'existence de l'exploitation et l'entretien des sols.

Le DPU peut être vendu. II est la propriété de l'exploitant et non du propriétaire. II peut même être vendu sans les terres. Mais le paiement ne peut être perçu que si la terre est entretenue. Il doit donc toujours être associé à une surface.

\section{Les effets probables}

Nous étudierons les effets de la mise en place de ce droit à paiement unique dans les décisions de gestion de l'exploitation agricole à court terme, puis à long terme. Pour faciliter la compréhension, nous effectuons nos raisonnements avec l'hypothèse d'un découplage à $100 \%$. Ceci n'est pas une prise de position partisane pour cette solution mais simplement un choix de technique de présentation. Le découplage à $75 \%$ entraîne les mêmes conséquences avec un effet amorti. II ne faut pas exclure qu'à long terme une nouvelle réforme amène l'ensemble des Etats à pratiquer un découplage total, même si au démarrage de la réforme ils ont choisi un découplage à $75 \%$.

- La création d'un résultat d'entretien ou d'une rémunération d'entretien des terres.Parmi les actuelles charges fixes et charges variables de l'exploitation, on peut trier celles qui sont liées au seul entretien des terres, et celles qui sont liées à l'acte de production. Alors que nous avions l'habitude de raisonner sur un résultat global de l'exploitation, on en vient à raisonner sur la rentabilité de l'exploitation au travers de la somme de deux éléments : le résultat (ou la rémunération) d'entretien [égal à l'aide découplée moins les charges variables et fixes liées à l'acte d'entretien] et le résultat de production égal aux ventes (et aux aides qui restent couplées à la production) moins les charges variables et fixes de production. Le résultat étant celui de l'entreprise. Suivant le niveau des prix et des aides couplées, la répartition du résultat actuel entre le résultat d'entretien et le résultat de production peut être extrêmement différente. Parfois, le résultat d'entretien est très nettement supérieur au résultat de production. Dans chaque exploitation, pour chaque production, et parfois pour 
chaque parcelle, cette analyse devra être faite pour comprendre les enjeux de la réforme dans la gestion de l'exploitation.Nous avons retenu ce mode de raisonnement parce qu'il correspond aux fondements de cette réforme justifiée dans l'argumentation de ses concepteurs par la multifonctionnalité de l'agriculture. Ce raisonnement suppose que les paiements uniques soient durables. S'ils ne l'étaient pas, il faudrait alors les considérer en termes de décisions de gestion de l'entreprise comme des financements à l'adaptation ou à la transformation de l'entreprise agricole.

- L'ordre des marges brutes est changé : vers de nouveaux choix d'emblavements ?Le paiement unique devenant une recette certaine, indépendante de la production, le calcul de la marge brute s'en trouve modifié. Il convient de calculer la marge brute sans les aides découplées. De ce fait, l'ordre des marges brutes de l'assolement peut se trouver profondément modifié. Les cultures non aidées avant 2003 voient leur niveau de marge mécaniquement réévalué.Par exemple, les calculs de marges réalisés dans la Marne montrent que la marge brute du blé est divisée par deux et que la marge brute de la betterave alcool de bouche et éthanol passe devant celle du blé.

- Rechercher l'EBE maximum hors DPU: le positionnement concurrentiel devient essentiel.En termes de gestion globale de l'entreprise, ces changements conduisent à maximiser l'EBE (excédent brut d'exploitation) sans les aides. Le positionnement sur le marché devient le fil rouge des choix de production de l'entreprise agricole. Dorénavant, les choix de production dépendront en priorité de l'espérance de gain par la commercialisation. C'est un changement majeur dans le raisonnement de gestion mais aussi dans le positionnement des acteurs économiques et dans la vision du métier des agriculteurs.Depuis 1936 pour le blé, depuis la création de l'Europe pour tous les produits soutenus par la PAC, l'agriculteur et la filière ont travaillé dans le cadre d'une sécurisation des marchés et des prix par les pouvoirs publics. Les décisions de gestion ont été essentiellement guidées par l'optimisation des facteurs internes de l'entreprise sans trop se soucier de la confrontation au marché.Depuis 1992 pour les grandes cultures et 1999 pour la viande bovine, les aides sont venues constituer un élément déterminant des recettes conditionné par l'acte de production. Le marché est intervenu pour une part plus importante dans la bonne tenue du chiffre d'affaires.Dorénavant, l'acte de production devra répondre avant tout à un marché. C'est bien d'une profonde mutation des éléments fondamentaux du métier qu'il s'agit.

- Vers une nouvelle gestion des facteurs de production.Le choix des productions n'est plus guidé par la chasse à la prime. La stratégie de l'entreprise doit gérer au mieux les ressources rares pour répondre à un marché solvable. Quelles vont être les ressources rares de demain compte tenu des différentes évolutions de la PAC mais aussi de la société européenne et des besoins fondamentaux d'une entreprise ? Ce seront :

- - le capital : c'est un élément fondamental, comme par le passé ;

- - la main-d'œuvre : c'est une ressource qui devient « rare » dans deux acceptions différentes : le coût de la main-d'œuvre salariée est élevé et celle qui est disponible est rare : c'est un des facteurs essentiels pour l'avenir ; mais la disponibilité, et plus encore l'intérêt et l'aspiration à de meilleures conditions de travail de l'exploitant luimême et de sa famille, prennent une importance majeure en ce début de siècle. Ils 
deviennent déterminants sur des actes de production comme la traite des vaches et l'irrigation du maïs ;

- - la quantité d'azote épandable : cette limite, dont l'application sera confortée par l'éco-conditionnalité des aides, pose le problème suivant: comment tirer le maximum de valeur ajoutée des unités d'azote qu'on peut épandre sur un hectare ? Par exemple, épandre de l'azote qui vient des vaches laitières permet-il de produire plus de valeur ajoutée que l'azote qui vient des taurillons?

- - l'eau d'irrigation: une autre gestion de l'eau verra le jour. Par exemple, si l'irrigation n'est plus une condition pour toucher un certain niveau d'aides, il pourra être préférable d'arroser du blé dans une phase critique que de réserver l'eau au maïs. Ceci sera renforcé par l'éco-conditionnaltié des aides de la PAC;

- - l'optimisation des contrats commerciaux et des quotas de production. Puisque les produits sont soumis à une plus grande variabilité de prix sur le marché, l'exploitant pourra donner priorité, dans son assolement, aux productions sous contrats ou dépendant d'un quota et bénéficiant par là soit d'une meilleure valorisation, soit d'une meilleure sécurisation.

\section{Le DPU : de l'aide au revenu à la gestion patrimoniale}

On l'a vu, les choix de production puis les décisions globales de gestion de l'exploitation s'affranchissent du montant du paiement unique. Toutefois, ils doivent respecter les critères d'écoconditionnalité. II se produit alors un glissement progressif de l'utilisation de ce paiement unique dans la vie et la stratégie de l'exploitant agricole.

- $\quad 1^{\text {ère }}$ étape : le soutien du revenu. A la mise en place du DPU, rien ne va changer si ce n'est la forme de l'aide. Son montant restera constant après la diminution due à la modulation. C'est la poursuite d'une aide au revenu. Et ce sera le cas tant qu'il n'y aura pas de changement notable du système d'exploitation.

- $-2^{\text {ème }}$ étape : le revenu d'entretien. Dès que la prise de conscience des nouveaux choix de gestion possibles sera faite, le paiement unique deviendra un financement du travail d'entretien du sol, avec ou sans production.

- $-3^{\text {ème }}$ étape : le moyen de financer des projets économiques ... ou personnels. Dans bon nombre de situations, l'exploitant va pouvoir arrêter ses productions habituelles et continuer à bénéficier d'un montant d'aides qu'il pourra utiliser pour investir dans d'autres projets agricoles ou même dans des activités économiques non agricoles. Un de ces projets pourrait d'ailleurs être une période de préretraite pendant laquelle le chef d'exploitation vivra du revenu d'entretien évoqué ci-dessus.

- $\quad-4^{\text {ème }}$ étape : le DPU contribue au patrimoine. Le montant du DPU, puisqu'il est vendable, va devenir un élément constitutif ou améliorant le patrimoine de l'exploitant.

En cela, il va faire évoluer profondément les pratiques, et probablement faudra-t-il faire évoluer le droit rural et réfléchir aux "politiques de structure » qui régissent le marché des exploitations 
agricoles et du foncier. En définitive, l'impact micro-économique des aides européennes devenues découplées quittera progressivement le domaine de l'économie de la production pour gagner celui de l'économie patrimoniale.

\section{Disjoindre la gestion patrimoniale et les décisions d'entreprise}

Dans les décisions de gestion et l'approche du droit rural actuel, les décisions de gestion de l'entreprise et les décisions de gestion patrimoniale sont intimement mêlées. Or, on observe depuis plusieurs années une élévation constante des prix du foncier et des pas de porte sur la plus grande partie du territoire. Celle-ci n'est pas justifiée par la rentabilité économique des exploitations qui a tendance à se tasser sérieusement. Cela veut dire que les agriculteurs sont prêts à mettre plus cher dans l'achat du foncier et des pas de portes que ne leur permet l'espérance de rentabilité. Au-delà d'une recherche de sécurisation de l'outil de travail, c'est une véritable stratégie patrimoniale qui se révèle dans cette augmentation des prix. Par ailleurs, le développement du tourisme, des résidences secondaires, de la rurbanisation, des loisirs vient concurrencer l'agriculture dans l'utilisation du territoire. II en résulte une augmentation de la valeur patrimoniale des biens ruraux qui se distingue de plus en plus de leur potentialité économique d'origine strictement agricole.

La logique de gestion patrimoniale de l'exploitation agricole était liée au travail et à la mise en production. Elle devient déliée du travail et de la mise en production. La mise en place du DPU va venir renforcer ce phénomène. A l'avenir, on aura donc à imaginer la gestion économique des entreprises agricoles de manière disjointe de la gestion patrimoniale des biens ruraux.

\section{Les effets de la réforme sur les productions et les territoires}

\section{Les grandes cultures}

\section{Un secteur économiquement affaibli}

La réforme intervient dans une filière grandes cultures française déjà affaiblie. Depuis la fin des années 90, les exploitations céréalières ont vécu une érosion forte de leurs revenus et de leur situation financière. Cette érosion est : moins forte dans les zones où la diversification a été faite en réaction à la PAC de 1992 ; la plus forte dans les régions à fort potentiel qui sont restées spécialisées dans les "Scop "; moins forte, mais touchant des revenus déjà beaucoup plus bas, dans les vastes zones intermédiaires.

Ces difficultés n'ont cependant pas entraîné de mouvement de restructuration de la production et le prix des reprises et du foncier n'a pas baissé... Cela veut-il dire que les exploitants pensent que la crise est passagère?

Parallèlement, les organismes stockeurs ont entamé un vaste mouvement de repositionnement sur les marchés qui se traduit par des investissements dans l'aval; une contractualisation des mises en culture; des démarches de qualité. Et de nombreuses restructurations ont déjà été faites. Elles diminuent rapidement le nombre de collecteurs de céréales et d'oléoprotéagineux. Néanmoins, les situations financières des organismes stockeurs sont parfois difficiles, gravement pénalisées de surcroît par la sécheresse de 2003. 


\section{Les effets de la réforme}

Les prix d'intervention étant maintenus par l'accord de Luxembourg, le secteur est essentiellement touché par le découplage. Les effets que nous décrivons ci-dessous seront bien entendu tempérés par le fait que la France choisira de maintenir $25 \%$ de couplage.

Les exploitants pourront dans l'absolu avoir trois options : produire, ne pas produire, faire produire.

- $-1^{\text {ère }}$ option : continuer à produire... autrement probablement. De nouvelles options vont être explorées telles que :

- - la priorité aux cultures qui laissent le plus de marge brute hors aide. Les cultures actuellement non aidées auront un regain d'intérêt. La variabilité inter-annuelle des emblavements augmentera avec la variabilité des prix ;

- - le choix d'itinéraire technique « low cost »;

- - une gestion différente des ressources en eau si on irrigue ;

- - une réduction des charges fixes, notamment par une nouvelle organisation du travail, une optimisation du matériel et une gestion collective des parcellaires.

- $\quad 2^{\text {ème }}$ option : ne pas produire. Un choix de non-production totale pourra être fait dans des situations un peu particulières qui resteront exceptionnelles: de fin de carrière ou de mauvais résultats techniques. Mais une gestion « à la parcelle » pourrait aussi voir le jour qui éliminerait la production sur les moins bonnes terres de l'exploitation pour des raisons d'éloignement ou de mauvaises qualités agronomiques par exemple. Les surfaces qui pourraient ainsi sortir de la production pourraient être significatives dans les zones intermédiaires.

- $-3^{\text {ème }}$ option : faire produire. Il s'agit de faire produire par un autre avec des degrés plus ou moins importants de sous-traitance, en gardant l'essentiel du bénéfice du paiement unique pour soi.

Cette situation pourra trouver à la fois des preneurs et des offreurs. Les offreurs : des exploitants en fin de carrière ; des héritiers qui veulent conserver le patrimoine; des pluri-actifs. Ils pourraient être nombreux. Les preneurs :

- - des exploitants bien équipés en matériel et disposant de main-d'œuvre qui n'arrivent pas à s'agrandir et qui doivent arrondir leur chiffre d'affaires et leur revenu. Ils sont nombreux. L’intérêt économique pour eux existe tant qu'ils travaillent à un coût marginal ;

- - des entreprises de travaux agricoles performantes et qui ont trouvé des modes d'organisation leur permettant de faire des offres de service à bas prix ou même des prises en charge totales de la culture à façon.

On voit que l'organisation même des exploitations et de la production pourrait être profondément modifiée. A terme, on pourrait voir se développer de nouvelles formes de faire-valoir associant l'exploitant en titre, souvent propriétaire, et le faiseur à façon dans une sorte de " néo-métayage ". 


\section{Les effets par zone}

On notera rapidement que :

- - dans les zones betteravières les évolutions ne pourront être imaginées que lorsque l'on connaîtra le nouveau règlement « sucre ";

- - dans les zones de grandes cultures diversifiées après 1992 l'impact du découplage devrait rester limité ;

- - dans les zones de grandes cultures non-diversifiées à fort potentiel tous les mécanismes évoqués vont jouer mais il n'y aura pas de surfaces non-cultivées en plus de la jachère obligatoire. Le travail à façon peut s'y développer ;

- - dans les zones intermédiaires tous les mécanismes vont jouer. La non-production y sera plus tentante qu'ailleurs. Les parcelles les moins bonnes seront placées hors production. Le mouvement de restructuration réel ou virtuel (travail à façon) pourrait être important.

Des zones non-cultivées pourraient voir le jour. Ceci sera freiné par un recouplage à $25 \%$.

\section{Les effets par production}

C'est le maïs qui devrait le plus souffrir de la nouvelle PAC, en particulier dans les zones irriguées et dans les zones d'élevage où il devrait diminuer au profit de l'herbe. Le blé tendre pourrait vivre un double mouvement de perte de surface liée à la non-production et d'augmentation de surface liée à la disparition des élevages dans les exploitations de polyculture. Dans un certain nombre de cas, il pourrait devenir un blé "d'occupation du territoire ", ce qui tendrait à limiter les rendements, et donc, les volumes. Le blé dur devrait régresser. La réussite passera par des démarches de qualité. Les oléoprotéagineux ne devraient pas être particulièrement touchés. Et le pois pourrait être en régression, comme le maïs dans les zones irriguées. Mais les arbitrages entre productions végétales seront beaucoup plus influencés que par le passé par la situation des marchés et les positions concurrentielles.

\section{Les effets globaux}

En résumé, avec une certaine progressivité, les effets sur les grandes cultures seront :

- - une réhabilitation des cultures actuellement non-aidées ;

- - une tendance à la diminution des surfaces de production et des volumes produits avec un effet sur l'utilisation des territoires ;

- - un mouvement de restructuration des exploitations à la fois réelle, virtuelle et industrielle ;

- - une restructuration au sens classique par l'augmentation de taille ;

- - une restructuration virtuelle par le développement de la prestation de services ;

- - une restructuration au sens industriel par l'externalisation d'une partie des moyens de production ; 
- - une diminution de l'utilisation des intrants créant des difficultés dans les entreprises d'amont. Cela débouchera sur des restructurations ;

- - un intérêt des producteurs pour la contractualisation et les démarches filières ;

- - une restructuration des organismes stockeurs dans des pôles pratiquant une forte dynamique de l'offre vers les utilisateurs finaux.

En outre, de nouvelles possibilités s'ouvrent aux cultures carburants. Elles devront faire l'objet de la plus grande attention.

\section{La production bovine}

Le choix, par la France, du maintien à $100 \%$ de la prime à la vache allaitante et à $40 \%$ de la prime d'abattage est fait. C'est sur cette base que nous étudions l'évolution de la production bovine en France qui, comme on le sait, fournit trois marchés très différents par leur nature et leur organisation : le marché du broutard, le marché européen de viande jeune et le marché français. Par ailleurs, l'élimination progressive des restitutions fera fondre la possibilité d'exportation de viande française hors Europe.

\section{Le troupeau allaitant}

En production allaitante, le choix du couplage à $100 \%$ entraîne pour l'exploitant une nécessité de poursuivre la production en place pour continuer à bénéficier des primes vaches allaitantes, indispensables à son système d'exploitation et à son revenu. II n'y aura pas de préretraite possible pour les producteurs de vaches allaitantes en prenant appui sur le découplage. Cela conduit ces exploitations et les vastes territoires concernés : à un maintien des systèmes de production en l'état ; à un maintien de l'utilisation de ces territoires par ces exploitations; à une forte tentation d'élimination de l'activité d'engraissement pour des raisons économiques et d'organisation du travail. Ceci pourrait limiter ou déstabiliser les efforts réalisés dans ce domaine pour structurer des filières de viande de qualité.

On notera le fort engouement actuel des producteurs de ces zones pour la production de broutards, moins exigeante en organisation et en quantité de travail que l'engraissement. Cet engouement repose sur un niveau de prix relativement élevé qui n'a pas toujours existé et dont le maintien n'est pas certain.

L'ensemble de la filière allaitante se trouvera encore plus soumis à la fluctuation de la demande des donneurs d'ordre italiens, espagnols, voire grecs.

En définitive, le choix du couplage des PMTVA (prime au maintien du troupeau de vaches allaitantes) à $100 \%$ stabilise et protège la situation actuelle. On prendra garde que ce choix ne constitue pas une sorte de ligne Maginot qui puisse être contournée par l'absence de structuration des filières et d'actions commerciales d'envergure !

\section{La production de taurillons}

La mise en production de lots de taurillons ne dépendra plus des aides. Elle résultera seulement d'un pari sur la rentabilité économique pour laquelle le prix d'achat des broutards, le prix espéré de vente 
en carcasse et les coûts de production seront les éléments décisionnels. Cela ne suffira plus pour permettre d'investir dans de nouveaux ateliers dans leur conception actuelle. Dans les ateliers existants, la production sera décidée lot par lot en fonction du niveau des cours. Il en résultera un problème d'approvisionnement des abattoirs spécialisés, en particulier dans le Grand Ouest. La poursuite de l'activité de cette filière nécessitera que les entreprises de la filière sécurisent au moins partiellement la rentabilité pour les producteurs. La filière sera tentée d'imaginer de nouvelles formes de production, diminuant les coûts et les contraintes. De nouvelles formes de contrats pourront voir le jour. Dans l'Ouest, les ateliers de taurillons, annexes à un atelier laitier, pourront régresser au profit d'un agrandissement de l'atelier laitier pour simplifier le travail.

\section{Les vaches de réforme}

Elles constituent, bon an mal an, l'essentiel de la consommation de viande du marché français. La disparition accélérée du métier de boucher dans les GMS renvoie le travail de la viande dans les ateliers des quelques grandes entreprises du secteur. Celles-ci vont se substituer aux bouchers pour déterminer la qualité de la carcasse. L'industrialisation de la découpe et du packaging diminuera l'influence du type d'animal. A la recherche d'une matière première à bas prix, ces industries privilégieront les vaches de réforme.

\section{La production laitière}

Le couperet de la baisse des prix d'intervention sur le beurre (- $25 \%)$ et sur la poudre (-15\%) vient cueillir la filière laitière à un moment où elle rencontre des difficultés importantes. Les laiteries françaises sont confrontées à un problème général d'excédent sur les marchés et, pour bon nombre d'entre elles, à un problème de rentabilité. Autant dire que ces problèmes vont être aggravés par cette baisse des prix d'intervention.

Les laiteries devront donc trouver des réponses qui vont s'articuler autour des axes suivants :

- - réduction des prix de revient internes ;

- - redéploiement des fabrications vers d'autres produits que le beurre et la poudre... avec un effet de domino ;

- - rationalisation de la collecte ;

- - pression sur le prix du lait ;

- - restructuration de la branche avec une diminution du nombre de sociétés et du nombre d'usines ;

- - recours à du lait provenant d'exploitations « qualifiées».

De leur côté, les exploitations laitières sont placées devant deux enjeux majeurs. La plupart d'entre elles n'ont pas procédé à la mise aux normes de leurs bâtiments d'élevage. Celle-ci devra être faite, entraînant mécaniquement une augmentation des prix de revient du litre de lait... au moment où le prix de vente de celui-ci a de bonnes chances de baisser. La faisabilité de cet investissement obligatoire se trouvera donc bien souvent remise en question. Par ailleurs, la mise en place des 35 heures est venue cristalliser, chez les producteurs, leur détermination à bénéficier de conditions 
de travail équivalentes à celles des autres actifs. Plus précisément, la volonté de sortir de l'astreinte de la traite est devenue une revendication et/ou un objectif personnel majeur. Bien souvent, la traite est faite par une femme de la génération du baby boom. La production laitière pourrait donc être remise en question avec le départ en retraite des « mamy boom » d’ici 2008-2010.

\section{Les effets de la baisse des prix d'intervention sur les exploitations}

Si ces effets ne sont pas mécaniques, il y a fort à parier qu'il y aura une tendance à la baisse du prix payé au producteur. Celle-ci entraînera :

- - un changement de l'attractivité du métier : il faut rappeler que le métier de producteur de lait a été construit sur une bonne sécurité du prix du lait. Celui-ci est devenu un repère fondamental de l'attractivité du métier. Le passage à une plus grande variabilité du prix et/ou à une période de baisse du prix pourrait avoir un effet dévastateur sur l'attractivité du métier, toutes générations confondues ;

- - une diminution de rentabilité des exploitations : la baisse de prix, probablement non totalement compensée par l'aide directe, va réduire la rentabilité des exploitations. Cela va déstabiliser financièrement les petites structures spécialisées, empêcher la mise aux normes d'un bon nombre d'exploitations et rendre non transmissibles les exploitations moyennes non modernisées.

Les régions ou départements à petites structures spécialisées seront plus gravement touchés.

Les effets de la mise en place d'une aide directe... découplable en 2007

Cette mise en place sur trois ans, suivi d'un découplage, va entraîner un double mouvement :

- $-1^{\mathrm{er}}$ mouvement : les producteurs de lait qui avaient un projet de transmission vont être tentés d'attendre que leur droit à paiement unique soit constitué. La période 2004-2007 va être une période d'attente freinant installations et restructurations.

- $\quad 2^{\mathrm{e}}$ mouvement : après 2007 , une fois le droit à paiement unique constitué, les exploitants seront libres des choix d'orientation de leurs productions. Des arrêts de production et de restructurations importants sont à prévoir à partir de cette date.

\section{Qui produira du lait demain ?}

Sous réserve que l'agriculteur puisse disposer d'une laiterie pour collecter et transformer son lait ce qui pourrait devenir une vraie question sur certains territoires -, le producteur et l'exploitation laitière pourraient avoir les profils suivants.

Avant tout, le producteur de lait sera un passionné du métier. En clair, la nouvelle PAC devrait inciter ceux qui ne croient pas, ne croient plus, au métier ou n'en n'ont plus envie, à le quitter. L'arrêt de production va concerner :

- - les ateliers laitiers dans les exploitations de polyculture élevage, en particulier dans les régions de grandes cultures. L'élimination de la production laitière se fera avec le départ des parents. La production dans les zones intermédiaires en sera affectée ; 
- - les ateliers laitiers non transmissibles appartenant à des exploitants de + de 50 ans qui trouveront une formule de transition avec un repreneur qui veut agrandir son propre outil de production. La demande de cessation-transmission dans cette situation est très importante ;

- - les ateliers laitiers qui pourront se reconvertir vers le troupeau allaitant un peu partout mais particulièrement en zone de montagne et en zone allaitante. Ce mouvement sera limité uniquement par le nombre de primes PMTVA disponibles !

Les passionnés vont spécialiser, agrandir ou restructurer leur atelier. Les producteurs passionnés, à la tête de petits ateliers, vont rechercher des solutions par l'augmentation de leur quota ou le rapprochement avec une autre exploitation pour résoudre à la fois la contrainte économique et la contrainte du travail. Les grandes exploitations viseront la spécialisation. Elles chercheront à éliminer les ateliers anciens tels que les taurillons ou certaines cultures pour simplifier le travail et régler des problèmes d'environnement. Parallèlement, elles chercheront à augmenter leur quota, en particulier pour se donner les moyens de se payer un remplacement hebdomadaire à la traite (par l'emploi d'un salarié ou par un robot).

Celles de ces exploitations qui ont déjà réalisé la mise aux normes dans de bonnes conditions de financement public seront très courtisées par les laiteries qui chercheront sans doute à se constituer un réseau de collecte performant. Le développement d'un dispositif contractuel entre les laiteries et leurs producteurs pourrait amener à envisager d'un autre œil le système actuel des quotas d'ici quelques années.

\section{Quels effets territoriaux ? Quelle évolution de la réglementation ?}

On vient de voir que la nouvelle PAC va entraîner un double mouvement de restructuration. Les exploitations vont se restructurer à partir de 2007 et les entreprises laitières vont se restructurer progressivement sur la période. Cette filière a donc besoin de mouvement. Or, le dispositif réglementaire actuel de gestion départementalisée des quotas, liés au foncier, fige la production dans le département. En outre, il fonctionne suivant un principe distributif. On s'en félicitera dans son effet bénéfique à maintenir les choses en place. Mais ce maintien a été économiquement possible parce que le prix du lait était au niveau que nous avons connu, permettant d'assurer le revenu par le prix avec les structures existantes. A un niveau nettement inférieur du prix du lait d'une part et avec un découplage de l'aide directe d'autre part, le cloisonnement départemental de la production et une politique distributive ne constituent plus des éléments suffisants de réussite de la production laitière sur un territoire. II faut que les laiteries et les exploitations réussissent chacunes à dégager un revenu et une rentabilité des investissements.

Si on vise l'avenir à long terme de la production laitière, il faut s'interroger sur les moyens à mettre en œuvre dans la réglementation pour préparer cet avenir. Autant que possible, il faudrait qu'un producteur passionné (ils ne seront pas si nombreux) puisse trouver des quotas pour financer ces investissements de mise aux normes, la rémunération de son remplacement hebdomadaire à la traite et sa propre rémunération tout en ayant baissé son prix de revient. Par ailleurs, une augmentation de la production devrait pouvoir se faire sans forcément augmenter la surface exploitée parce que ce n'est pas forcément nécessaire économiquement. Aussi, convient-il de 
s'interroger sur l'opportunité de maintenir un lien strict de la gestion des quotas par rapport au foncier et dans un cadre départemental, et sur l'efficacité de maintenir une politique distributive des quotas.

\section{Les pistes pour l'agriculture de demain}

\section{Regarder le changement dans les yeux}

Deux dangers sont à éviter : regarder dans le rétroviseur et cultiver le franco-français.

- Eviter de regarder dans le rétroviseur. Parce que la nouvelle PAC perturbe radicalement les archétypes, les raisonnements et les intérêts économiques immédiats, la tentation est grande de chercher avant tout à ralentir et à neutraliser les changements avec l'espoir de conserver le maximum d'acquis. Ainsi, il pourrait être tentant d'utiliser les nouveaux outils de la PAC dans l'esprit des lois de 1962. Aujourd'hui, les changements dans le contexte mondial, européen, politique et social sont tels que cette attitude pourrait se retourner à moyen terme contre le positionnement concurrentiel des agriculteurs et des entreprises agroalimentaires françaises, avec un effet boomerang désastreux pour les territoires. En effet, le nouveau contexte oblige à placer la réussite concurrentielle de nos filières au cœur de la stratégie nouvelle. Les aides, toujours nécessaires, et les interventions publiques, qui restent indispensables, ne peuvent plus être le fondement des décisions économiques des acteurs. Elles doivent en devenir l'accompagnateur.

- Eviter le franco-français. On sait combien la nouvelle PAC ouvre des possibilités à la renationalisation des politiques agricoles. La France a souvent la tentation de se donner des règles spécifiques (référentiel agro-bio - normes environnementales...) souvent plus exigeantes que la norme européenne. Or, le marché et la concurrence sont toujours européens et souvent mondiaux. Trop de spécificités peut conduire à diminuer notre capacité concurrentielle. Il faut veiller à ce que nos propres règles nationales soient établies dorénavant en ayant intégré la réalité de l'élargissement du marché et de la concurrence au niveau de l'Europe : gardons-nous de trop de spécificités réglementaires !

- Eviter le flou et les incohérences. Pour construire leur avenir, les personnes et les entreprises ont besoin de connaître les règles du jeu. C'est particulièrement le cas en agriculture où les investissements s'amortissent sur une longue durée. Aussi est-il souhaitable de clarifier les règles et de dire où on veut aller. Immédiatement, cela a deux types d'application : attention à la longueur de la période de transition : plus la transition sera longue, plus elle créera de cas particuliers et de complications administratives, et surtout, plus elle risque de retarder des prises de conscience et des prises de décisions.

Attention à la cohérence des dispositifs : c'est aujourd'hui à deux endroits que les opérateurs économiques ont besoin de cohérence. Au niveau européen, il est urgent que les règles d'importation des produits et les règles d'étiquetage soient mises en cohérence avec les contraintes imposées à la production à l'intérieur de l'Europe. Il faut établir une cohérence entre les choix concernant la défense du consommateur, l'environnement et le commerce international. Au niveau français, il faudra là aussi rétablir une cohérence entre la réglementation européenne, les besoins de gestion des entreprises agricoles et le droit rural. 


\section{Construire sur les nouvelles demandes de la société... vers la réussite par la dynamique de l'offre}

On le sait, le marché des besoins alimentaires de base, pour lequel a été construite notre agriculture à partir de 1960, est soumis à une inexorable pression à la baisse des prix. A côté, d'autres marchés émergent pour lesquels l'agriculture française a les moyens de relever le défi. Citons entre autres : la production de bio-carburants (la nouvelle PAC ouvre de nouvelles possibilités); la production de matières pour l'industrie non alimentaire ; le formidable marché touristique français... quasiment délaissé par l'agriculture française ; l'agro-biologie ; les nouvelles attentes diététiques; la réponse aux attentes sécuritaires dans l'alimentation ; la réponse aux attentes du consommateur sur le goût et la praticité ; la place que l'évolution de l'urbanisation et des NTIC ouvre à de nouvelles formes de distribution.

Dans tous les cas, le passage d'une vision d'une agriculture tournée vers la production à une agriculture centrée sur le produit-service sera déterminant. Dans tous les cas aussi, c'est la dynamique de l'offre qui sera déterminante.

\section{La réussite par la dynamique et l'organisation en filière... vers la contractualisation}

L'abaissement des filets de sécurité européens sur la gestion des prix et des volumes ramène l'agriculture dans le chemin classique de la réussite économique en milieu concurrentiel. Les IAA et les exploitations agricoles sont donc conduites à coopérer dans une dynamique de filière permettant de réussir sur le marché. La mise en œuvre des initiatives, la prise de risque et le partage de la valeur ajoutée devront faire l'objet d'un partenariat, de négociations et de contractualisations généralisés.

Tout ceci devra être conduit en privilégiant le partenariat à long terme qui est seul compatible avec la lourdeur des investissements que nécessite l'agriculture. En la matière, une évolution des mentalités et des pratiques de partenariat est indispensable.

\section{Remplacer la sécurisation publique des marchés et des prix... vers une gestion privée du risque}

Il est nécessaire de gérer dorénavant les exploitations agricoles dans un contexte de variabilité des prix et des marchés qui ne sont plus sécurisés par la puissance publique européenne. Pour cela, il faut : augmenter la marge de sécurité interne de l'entreprise agricole. Cela veut dire, par exemple, que pour les exploitations laitières, les installations avec de petits quotas deviennent plus dangereuses; s'appuyer sur des démarches de qualité favorisant l'accès au marché ; développer I'utilisation de nouveaux outils que sont les contrats à terme et les assurances-revenus; construire une gestion interprofessionnelle des filières afin d'éviter de faire s'effondrer les marchés par des surproductions conjoncturelles ou structurelles.

\section{Améliorer la compétitivité... vers une nouvelle baisse des prix de revient}

Quels que soient les segments de marchés sur lesquels on veut vendre les produits agricoles français, la réussite passera par l'adaptation au marché évoquée ci-dessus mais aussi par une compétitivité en termes de prix. Ces deux éléments ne s'opposent pas. Ils sont continuellement associés dans la réussite. La recherche de compétitivité nécessitera de trouver les moyens de baisser les prix de revient. Beaucoup a déjà été fait sur les intrants depuis 1992. Mais il faudra encore trouver d'autres moyens de baisser les prix. Il faudra donc accepter d'ouvrir la réflexion sur: la structure des exploitations (sur ce point, la pyramide des âges ouvre des perspectives de restructuration) ; le poids 
des coûts de mécanisation; l'ensemble des charges fixes. Ces éléments ne pourront trouver de solution s'ils sont pris simplement un à un. Il conviendra de rechercher des solutions dans une évolution globale du système d'exploitation.

Cette question est d'autant plus importante à aborder si on entend maintenir des débouchés à l'export sur pays tiers avec les nouvelles contraintes de l'OMC qui vont vers la suppression des restitutions.

\section{Conjuguer le respect de l'environnement et la rentabilité... vers l'agriculture de précision et l'hypertechnicité du vivant}

Les voies anciennes d'intensification doivent être reconsidérées au regard des nouvelles exigences de la société qui sont traduites dans les critères d'éco-conditionnalité des aides. En même temps, la réussite ne passe plus par un soutien public des prix. II faut donc conjuguer le respect de l'environnement et la rentabilité.

L'extensification pourra être explorée dans quelques situations. Surtout, il y aura lieu de reconsidérer le choix du niveau de couverture du risque retenu dans la conduite de l'exploitation et des productions agricoles. Plus généralement, c'est l'agriculture de précision et l'utilisation approfondie des techniques du vivant qui vont constituer la double voie de l'évolution des systèmes de production. Loin d'aller vers un retour à l'agriculture de " grand papa " sur laquelle certains font parfois des rêves régressifs, l'avenir de l'agriculture française se construira à partir d'une hypertechnicité du vivant.

\section{Conjuguer rentabilité, organisation du travail et progrès social... vers l'externalisation et le travail en réseau}

Les attentes des agriculteurs en termes de conditions de travail d'une part, et les exigences de baisse des coûts de production d'autre part, vont conduire à une remise en cause de la conception actuelle de l'entreprise agricole et de l'exercice du métier. Jusqu'à aujourd'hui, l'exploitant a en charge la réalisation de toutes les fonctions de l'entreprise, de toutes les tâches de production et d'administration. De même, il doit être équipé pour l'ensemble de celles-ci. Une voie s'ouvre avec la nouvelle PAC pour explorer en agriculture les voies qui ont réussi en industrie.

Demain, l'organisation du travail et des investissements pourra être construite autour: de la réalisation par l'exploitant et la main-d'œuvre de l'exploitation des tâches dans lesquelles ils sont les plus compétents et les plus efficaces, et de l'externalisation des tâches pour lesquelles ils sont moins efficaces. De même, les investissements pourront être réalisés à plusieurs; les parcelles, gérées à plusieurs. Le travail à façon pourra être une solution intéressante pour le donneur d'ordre et le réalisateur. Demain, l'exploitation agricole ne sera plus conçue et gérée de manière autarcique. Les constituants n'en seront plus pensés comme des éléments figés sur une carrière.

Demain, l'exploitation agricole sera une entité économique souple et adaptable dans le temps en fonction du contexte et des contrats. Cette flexibilité touchera tout à la fois la surface et son utilisation, la main-d'œuvre, le capital et les investissements. Le travail en réseau, la flexibilité d'organisation de l'entreprise et la sous-traitance vont devenir des éléments forts du métier. 


\section{Conjuguer la nouvelle PAC et notre droit rural... vers un fonds agricole et une nouvelle politique des structures}

Il faut souligner la cohérence qu'il y a eu entre la volonté de répartir équitablement les moyens de production au moyen d'une politique distributive des structures et l'ancienne Pac qui garantissait à peu près les revenus au travers d'une garantie de prix. Ceci rendait possible cela. Dorénavant, deux éléments changent. D'une part, il n'y a plus une garantie de prix permettant une garantie de revenu. Le revenu doit venir, au-delà des aides toujours nécessaires mais insuffisantes, d'une réussite par le marché. D'autre part, la nouvelle PAC crée un droit à paiement unique attaché avant tout à l'exploitant qui peut le vendre. La France devra remettre de la cohérence entre ces éléments fondamentaux en prenant bien en compte les éléments évoqués ci-dessus.

En conséquence, il faudra explorer: la création d'un véritable "fonds agricole " et la mise en cohérence de la pratique et du droit concernant le nouveau bien que constitue le paiement unique et la manière dont sont gérés actuellement les droits à produire.

Les nouvelles solutions devront permettre à l'agriculture française de se préparer pour demain, c'està-dire se préparer à affronter la concurrence. On se tromperait à maintenir les choses en l'état pour figer l'activité immédiate sur chaque territoire. Le risque d'une sorte de glaciation de la production serait de conduire à une perte irréparable de compétitivité sur le long terme. La politique des structures doit être réévaluée au regard de cette contrainte de compétitivité.

\section{Conjuguer la compétitivité et l'activité sur les territoires... vers la dynamique de projet et I'utilisation du deuxième pilier}

Longtemps, le maintien de l'activité agricole sur les territoires a été assuré par les filets de protection européens sur les prix agricoles. Aujourd'hui, ceci disparaît avec le découplage, hormis pour le troupeau allaitant puisque la France a choisi de maintenir le couplage. Les territoires vont voir évoluer leur agriculture. Les mauvaises terres pourront être délaissées. Les acteurs agricoles ne seront plus seulement des exploitants professionnels mais aussi des préretraités, des pluri-actifs et des détenteurs de droits patrimoniaux. Les activités économiques pourront évoluer tant au niveau de la production que de l'amont et de l'aval.

On a souligné l'importance majeure qu'auront les dynamiques de filières. Cette dynamique va s'élaborer à partir des atouts et des contraintes du territoire non seulement dans un sens strictement agricole (aspects pédo-climatiques, compétence des agriculteurs, structures...) mais aussi dans des aspects non agricoles essentiels (population consommatrice, image, transport, urbanisme, tourisme...). Et avant tout, cette dynamique dépendra des initiatives, de la créativité et de la prise de risques des acteurs privés.

Ce contexte amènera les collectivités à appuyer ces initiatives dans les différents domaines que nous venons de citer. C'est pourquoi il est temps que la France apprenne à utiliser pleinement l'ensemble des dispositifs d'appuis européens. II faudra utiliser au mieux les fonds du deuxième pilier pour financer les projets d'adaptation au marché ou d'adaptation de l'appareil de production au nouveau contexte. 


\section{Conclusion}

En définitive, tout se passe comme si l'agriculture française se trouvait placée devant l'obligation d'accepter une transformation profonde de son contexte de travail. Le contexte international a changé. Le marché mondial a changé. Le marché européen change. La société change. Le changement de PAC vient traduire plus ou moins bien ces changements.

Tout cela met fin aux visions précédentes de la place de l'agriculture dans la société. En 1960, l'agriculture a eu pour mission de nourrir les français et les européens. En 1980, on a dit à l'agriculture qu'elle devait être le "pétrole » de la France, c'est-à-dire une source de devises. En 2004, les citoyens français et européens attendent avant tout de l'agriculture qu'elle les aide à vivre longtemps en leur fournissant de bons produits, dans un beau paysage... Le marché guide. L'Etat apporte aux producteurs un soutien socialement acceptable avec un appui dans les situations difficiles. II en résulte la nécessité de : franchir une nouvelle étape dans la performance économique des exploitations qui nécessite de renouveler nos schémas actuels (structures, technique, organisation...); construire une autre image intérieure du métier d'agriculteur, dans ses fonctions, dans ses techniques et dans la prise de décisions; construire une nouvelle vision de l'entreprise agricole dans son périmètre, dans sa dynamique et dans les choix qui la structure; construire de nouvelles relations économiques entre les partenaires d'une filière dont la réussite commune passe obligatoirement par la satisfaction d'un client sur un marché ; construire une nouvelle politique d'accompagnement de l'agriculteur par la puissance publique qui quitte la voie de la logique distributive pour passer au soutien du développement de projet.

Peut-être faut-il voir la nouvelle PAC comme la signature de l'acte de décès de l'ancienne vision que nous avons eu de l'agriculture. En cela, elle nécessite un processus de deuil... Aujourd'hui, l'heure est à l'élaboration d'un nouveau projet pour l'agriculture. Les choix qui en découlent ont probablement autant d'importance que les choix des années 1960 . On se rappelle l'audace des responsables de cette époque, leur clairvoyance et le courage qu'ils ont eu pour rompre avec le passé. II s'agit de rééditer leur formidable exploit et oser inventer une autre agriculture française. 
En $€$ constants de 2002

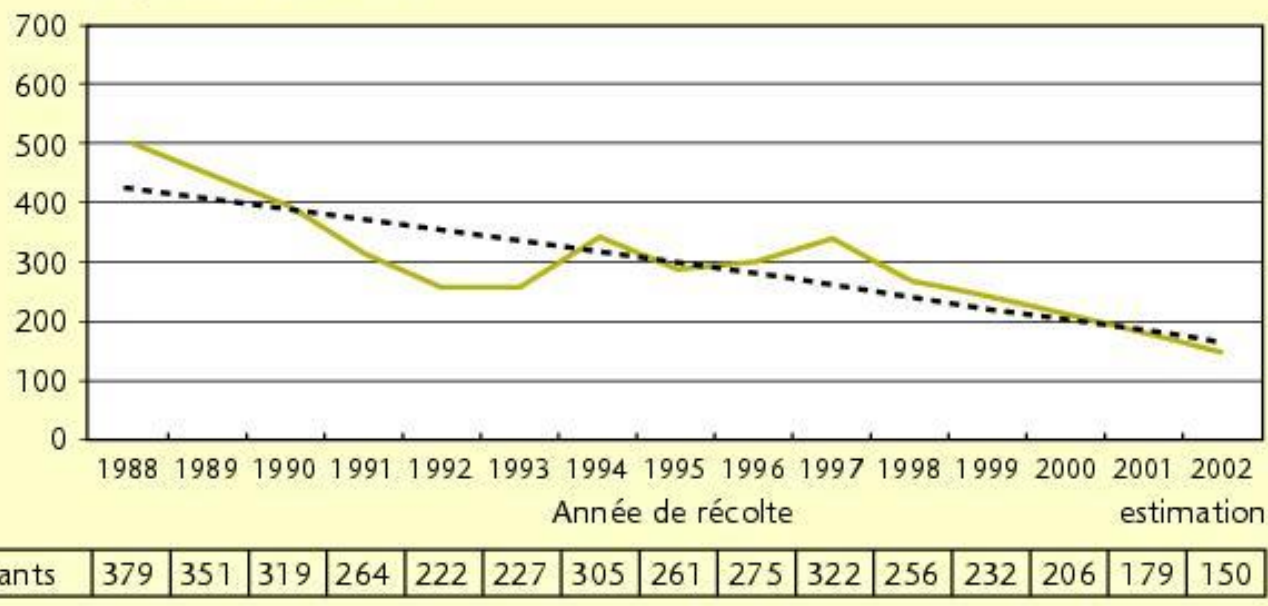

Figure 1 Evolution du résultat courant en Nogentais.

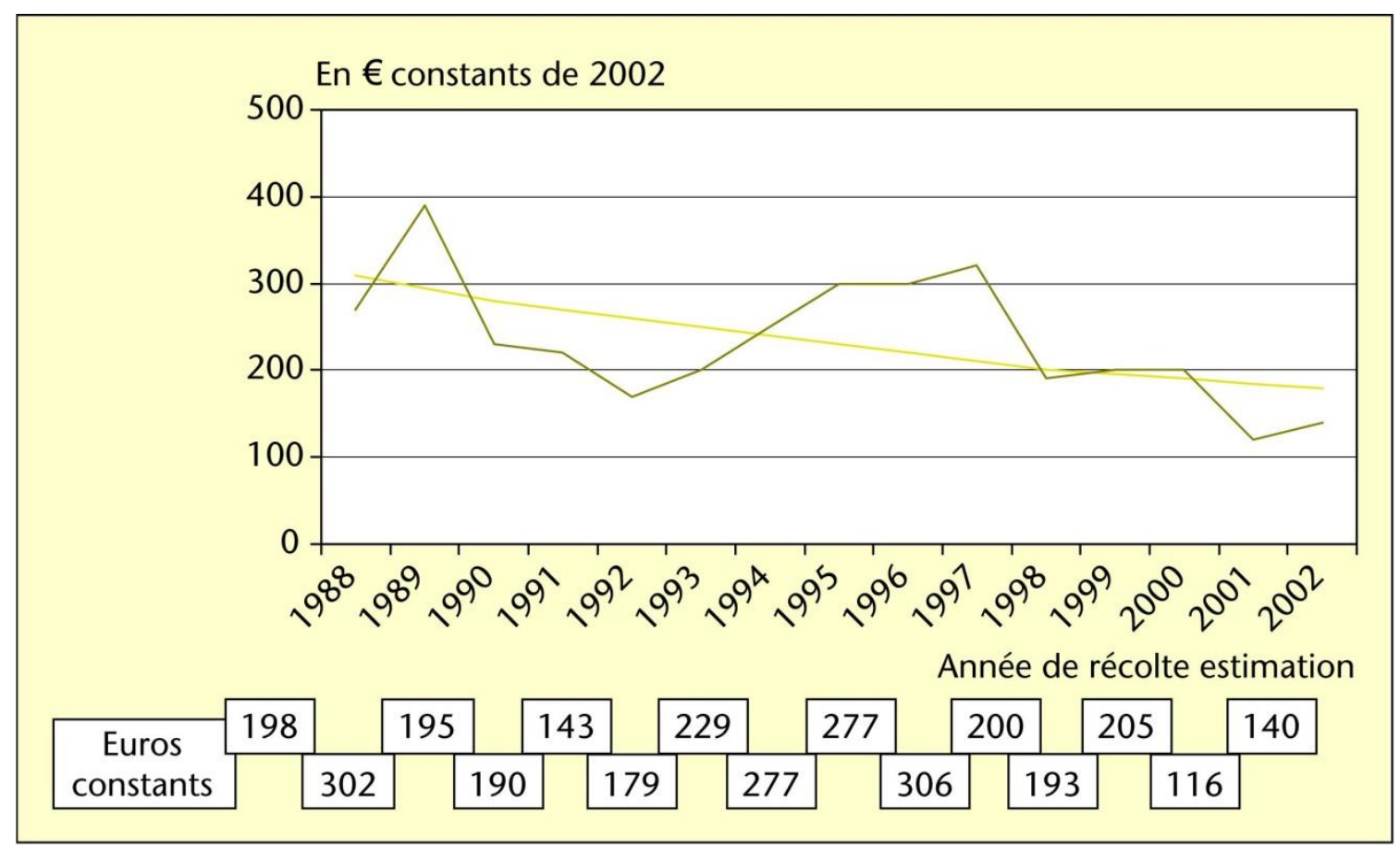

Figure 2 Evolution du résultat courant en Barrois. 